\title{
Strategies for an Efficient Official Publicity Campaign
}

\author{
Juan Neirotti ${ }^{1}$ \\ Received: 11 January 2021 / Accepted: 16 April 2021 / Published online: 29 April 2021 \\ (c) The Author(s) 2021
}

\begin{abstract}
We consider the process of opinion formation, in a society where there is a set of rules $B$ that indicates whether a social instance is acceptable. Public opinion is formed by the integration of the voters' attitudes which can be either conservative (mostly in agreement with $B$ ) or liberal (mostly in disagreement with $B$ and in agreement with peer voters). These attitudes are represented by stable fixed points in the phase space of the system. In this article we study the properties of a perturbative term, mimicking the effects of a publicity campaign, that pushes the system from the basin of attraction of the liberal fixed point into the basin of the conservative point, when both fixed points are equally likely.
\end{abstract}

Keywords Online learning $\cdot$ Disordered systems $\cdot$ Sociophysics

\section{Introduction}

In the present article we analyze the effects that publicity campaigns have on the opinionformation process in a population of interacting agents. We assume that the agents, or voters, live in a working society, i.e. a society in which there exists a set of rules $B$ that determine the acceptability of a social issue. $B$ can be thought as laws, social conventions, or otherwise that fix a reference for what is considered normal social behavior [1-3]. $B$ also represents what we call society's official position.

Opinions are highly dynamic mental representations of individuals' beliefs, resulting from processes of inference frequently done with insufficient information. They play a fundamental role in individuals' reaction to social situations that can trigger collective responses [4-6]. In our model each voter, or agent, is provided with a simple neural network that allows it to learn from the social reference $B$ and from other peers. Peer interaction occurs between connected agents; the information about these connections is stored into a directed graph that determines the topology of the society [7-9]. If this interaction is sufficiently strong, local consensuses, opposed to the rule $B$ may appear. The combination of these two sources of disorder, introduced through the set of examples for the learning process, and through the

Communicated by Deepak Dhar.

$凶$ Juan Neirotti

j.p.neirotti@aston.ac.uk

1 Department of Mathematics, Aston University, The Aston Triangle, Birmingham B4 7ET, UK 
graph that fixes the social topology, produce a very exciting model with potential predictive capabilities.

There are several studies on the effects external influences exert on the opinion formation process in voters models $[10,11]$, in particular in $[12,13]$ it is concluded that the most effective strategy for budget allocation in advert campaigns is periodic in nature. Following that lead we will propose to model an advert campaign with a periodic perturbation.

By modeling a publicity campaign using a periodic perturbation, we can analyze the strategy (represented by the amplitude and frequency of the perturbation) is most adequate to change the public opinion in favor of the official position. The relevance of the present studies can be easily exemplified. The campaign for the 2016 UK referendum was based on incomplete or unreachable information: internal polls showed that $85 \%$ of the British population wanted more information from the Government. It also consumed vast amounts of resources (Vote Leave, the official leave campaign, obtained the right to spend up to $£ 7,000,000$, a free mailshot, TV broadcasts and $£ 600,000$ in public funds, whereas the official position of Government was backed by a $£ 9,300,000$ campaign [14]), and produced immediate effects. Understanding a process that consumes this quantity of resources is paramount.

The model we present in this article differs from the classical Deffuant model of opinion dynamics [15], where the interaction between voters takes place if the difference between the variables that indicate the opinions of the interacting agents is bellow a given threshold (bounded confidence model). In our model the interaction is between connected neighbors that learn to have an opinion on socials instances presented to them and classified as acceptable or not by a social rule $B$. The stronger the connection the larger the influence peers have in the local neighborhood, opening the possibility for the emergence of local consensuses opposed to $B$ (the details are presented in Sect. 2).

The article is organized as follows: In Sect. 2 we describe the general setting of the system. In Sect. 3 we present numerical results obtained from the application of the formulae described in Sect. 2, and in Sect. 4 we present our discussion and final considerations.

\section{The Model}

The rule $B$ and the agents $\{a\}_{a=1}^{M}$ classify binary strings $\mathbf{S} \in\{ \pm 1\}^{N}$ that are presented to them with binary labels $\sigma_{B} \in\{ \pm 1\}$ and $\left\{\sigma_{a} \in\{ \pm 1\}\right\}_{a=1}^{M}$ respectively. They do so by using a simple neural network. In order to balance the level of sophistication of the model with its analytical tractability, we provided the agents and $B$ with a perceptron [16]. Each perceptron is characterized by an internal representation vector $\left(\mathbf{B} \in \mathbb{R}^{N}\right.$ for the rule $B$, and $\mathbf{J}_{a} \in \mathbb{R}^{N}$ for the agents) such that the labels become $\sigma_{B}(\mathbf{S})=\operatorname{sgn}(\mathbf{B} \cdot \mathbf{S})$ and $\sigma_{a}(\mathbf{S})=\operatorname{sgn}\left(\mathbf{J}_{a} \cdot \mathbf{S}\right)$, where $\mathbf{V} \cdot \mathbf{S} \equiv \sum_{i=1}^{N} V_{i} S_{i}$ for all $\mathbf{V} \in \mathbb{R}^{N}$, and $\operatorname{sgn}(x)=1$ if $x>0,-1$ if $x<0$, and 0 if $x=0$. In the proposed scenario we consider all internal representations, $\mathbf{B}$ and all $\mathbf{J}_{a}$, to be plastic (i.e. they change over time).

The connectivity of the system is given by the graph $\boldsymbol{G}=\left\{\{a\},\left\{\eta_{a, b}\right\}\right\}$ where $\{a\}$ is a set of vertexes associated with the agents and $\left\{\eta_{a, b}\right\}$ is a set of strengths $\eta_{a, b}$ that represent the influence of agent $b$ on agent $a$. In this work we will only consider real, non-negative values of strengths $\left(0 \leq \eta_{a, b} \in \mathbb{R}\right)$. In the language of disordered systems strengths are quenched variables that are drawn from a suitable probability distribution (the particular distribution we used in this investigation is presented after Eq. (23)). The neighborhood of $a$ is defined as $\mathbb{N}_{a}=\left\{c \in[M]: \eta_{a, c}>0\right\}$ [17]. The total number of vertexes (or agents) is $M$, and the average neighborhood size is defined as: 


$$
v \equiv \frac{1}{M} \sum_{a=1}^{M}\left|\mathbb{N}_{a}\right|,
$$

where $\left|\mathbb{N}_{a}\right|$ is the cardinality of the set $\mathbb{N}_{a}$. We will consider graphs with $v \sim O(1)$ only. We say a bond $(a, b)$ is active if $\eta_{a, b}>0$ and passive otherwise.

\subsection{Update Algorithms}

Assuming that the population of interacting agents receives information taken from the set $\mathbb{S} \equiv\left\{\left(\sigma_{B, n}, \sigma_{\mathbb{N}_{a}, n}, \mathbf{S}_{n}\right), n=1, \ldots, T\right\}$, where the string $\mathbf{S}_{n}$ is presented at time $n$ and then discarded, $\sigma_{B, n}=\operatorname{sgn}\left(\mathbf{B}_{n} \cdot \mathbf{S}_{n}\right)$ and $\sigma_{\mathbb{N}_{a}, n}=\left\{\operatorname{sgn}\left(\mathbf{J}_{c, n} \cdot \mathbf{S}_{n}\right): c \in \mathbb{N}_{a}\right\}$ is the set form by the labels given by agent $a$ 's neighbors, the update equation for the internal representation of $a$ is:

$$
\mathbf{J}_{a, n+1}=\mathbf{J}_{a, n}+\psi_{a, n} \frac{\sigma_{B, n} \mathbf{S}_{n}}{\sqrt{N}}
$$

where $\sigma_{B} \mathbf{S} / \sqrt{N}$ is the (unit length) Hebb vector [18], that indicates the direction $\mathbf{J}_{a}$ must grow to imitate $B$ and $\psi_{a, n}$ is the learning amplitude, that regulates how the information is incorporated in the internal representation of $a$. The length of the opinion formation process $T$ is considered to be proportional to the length $(N)$ of examples presented to the agents. The structure we propose for the learning amplitude $\psi_{a}$ is the following:

$$
\psi_{a, n} \equiv f_{n} \frac{\left|\mathbf{J}_{a, n}\right|}{\sqrt{N}} \Psi_{a, n},
$$

where $f_{n}$ is a decaying function of $n$ and it implements the annealing of the learning process [19], $\left|\mathbf{J}_{a}\right| / \sqrt{N}=\sqrt{\sum_{j=1}^{N} J_{a, j}^{2} / N}$ is a scaling factor that has no impact on the learning efficiency of the algorithm [20] and it has been only considered for technical purposes, and the factor $\Psi_{a}$ carries information on the interaction of agent $a$ with the rule $B$ and with the neighbors $c \in \mathbb{N}_{a}$. To construct this factor we consider a mechanism of corroboration with peers [21-23], that works in the following way: if the opinion of $a$ on the current social issue $\sigma_{a}$ is identical to socially accepted position $\sigma_{B}\left(\sigma_{a} \sigma_{B}=1\right)$ the change proposed in (2) should produce an internal representation $\mathbf{J}_{a}$ closer in direction to $\mathbf{B}$, whereas if the agent and the social rule disagree $\left(\sigma_{a} \sigma_{B}=-1\right)$ and if the collective influence of the agreeing neighbors of $a\left(c \in \mathbb{N}_{a}\right.$ and $\left.\sigma_{a} \sigma_{c}=1\right)$ is sufficiently strong, then the modification proposed in (2) should make $\mathbf{J}_{a}$ grow opposite to $\mathbf{B}$. Thus we propose the following factor:

$$
\Psi_{a} \equiv 1-\Theta\left(-\sigma_{B} \sigma_{a}\right) \sum_{c \in \mathbb{N}_{a}} \eta_{a, c} \Theta\left(\sigma_{a} \sigma_{c}\right),
$$

where $\Theta(x)=1$ if $x>0$ and 0 otherwise is the Heaviside step function.

The learning algorithm (4) works in the following way: In the absence of any inter-agent interaction the agents $\{a\}$ learn from $B$ only, and their internal representations $\left\{\mathbf{J}_{a}\right\}$ grow in the direction of $\mathbf{B}$. This accounts for the first term of the right-hand-side of Eq. (4). But disagreement between agent $a$ and rule $B$ may arise, i.e. $\Theta\left(-\sigma_{a}(\mathbf{S}) \sigma_{B}(\mathbf{S})\right)>0$. In such cases there is a psychological cost for dissenting [24] which triggers the need in the agent to corroborate information with their peers [23]. If $\sigma_{a}(\mathbf{S}) \neq \sigma_{B}(\mathbf{S})$, the agent $a$ checks with their closest peers $c$ (where close refers to a criterion extracted from social impact theory $[25,26])$, whether $\sigma_{a}(\mathbf{S})=\sigma_{c}(\mathbf{S})$ or not. If the integrated contribution of agreeing neighbors 
is sufficiently large (second contribution at the right-hand side of (4)), $\Psi_{a}$ becomes negative and the internal representation vector $\mathbf{J}_{a}$ grows opposite to $\mathbf{B}$.

Let us define the unit vectors $\boldsymbol{b} \equiv|\mathbf{B}|^{-1} \mathbf{B}$ in the direction of the internal representation of $B, \boldsymbol{j}_{a} \equiv\left|\mathbf{J}_{a}\right|^{-1} \mathbf{J}_{a}$ in the direction of the internal representation of agent $a$ and $\boldsymbol{j}_{a, \perp}=$ $\left[1-\left(\boldsymbol{j}_{a} \cdot \boldsymbol{b}\right)^{2}\right]^{-1 / 2}\left[\boldsymbol{j}_{a}-\left(\boldsymbol{j}_{a} \cdot \boldsymbol{b}\right) \boldsymbol{b}\right]$ in the direction of the component of $\mathbf{J}_{a}$ perpendicular to B. Given that an agent's classification is obtained through information processing using the internal representation vector $\mathbf{J}_{a}$, and that any modification to the vector $\mathbf{B}$ in the direction of $\mathbf{B}$ does not produce any change on $B$ 's classifications, we will construct the update algorithm for $B$ by considering the vector:

$$
\mathbf{L} \equiv \frac{1}{M} \sum_{c=1}^{M} \boldsymbol{j}_{c, \perp}
$$

which is the arithmetic average over all the components of the internal representations $\mathbf{J}_{c}$ perpendicular to $\mathbf{B}$. Observe that $\mathbf{B} \cdot \mathbf{L}=0$. Then:

$$
\mathbf{B}_{n+1}=\mathbf{B}_{n}+\frac{\lambda_{o}}{\sqrt{N}} f_{n} \mathbf{L}_{n},
$$

where $\lambda_{o} / \sqrt{N}$ is a suitable scale factor. Observe that if $\lambda_{o} \sim O(1)$ the updates of $\mathbf{B}$ at each time step are very small, thus $\lambda_{o} / \sqrt{N}$ is a measure of the inverse inertia (if the mass of $B$ is infinite we wouldn't expect any change at all, thus $\lambda_{o}=0$ ). Observe also that $\left|\mathbf{B}_{n+1}\right|^{2}=\left|\mathbf{B}_{n}\right|^{2}+O\left(f_{n}^{2} N^{-1}\right)$, which implies that the length of the vector $\mathbf{B}$ does not change with the update. In Eq. (6) the social rule is slightly modified through a wisdom-of-the-crowd effect.

To help describe the state of the system we define the variables:

$$
\begin{aligned}
\phi_{a} & \equiv \sigma_{B} \boldsymbol{j}_{a} \cdot \mathbf{S} \\
\beta & \equiv \sigma_{B} \boldsymbol{b} \cdot \mathbf{S}
\end{aligned}
$$

and parameters:

$$
\begin{aligned}
R_{a} & \equiv \boldsymbol{j}_{a} \cdot \boldsymbol{b} \\
W_{a, b} & \equiv \boldsymbol{j}_{a} \cdot \boldsymbol{j}_{b} \\
Y_{a, b} & \equiv \boldsymbol{j}_{a, \perp} \cdot \boldsymbol{j}_{b, \perp} .
\end{aligned}
$$

The variables depend explicitly on the information $\left\{\sigma_{B}, \mathbf{S}\right\}$ whereas the parameters depend on the internal representations $\left\{\left\{\mathbf{J}_{a}\right\}, \mathbf{B}\right\}$ only. The variable $\beta$ is positive or zero and the smaller the $\beta(\mathbf{S})$ the higher the likelihood of $\mathbf{S}$ to be in the classification boundary (given by $\mathbf{B} \cdot \mathbf{S}=0$ ). The variable $\phi_{a}(\mathbf{S})$ indicates how much the vector $\mathbf{J}_{a}$ has to be modified to agree with $\mathbf{B}$. If $\phi_{a} \gg 0$ the modification needed is negligible, if $\phi_{a} \simeq 0$ the modification needed is moderated and if $\phi_{a} \ll 0$ the modification needed is substantial. In this context agreement means that the chances of two different agents or agent and social rule, assign the same classification to a given string $\mathbf{S}$ are larger than one half. The parameter $R_{a}$ represents the level of agreement of agent $a$ with $B, W_{a, b}$ represents the level of agreement between agents $a$ and $b$ and the parameter $Y_{a, b}$ represents the level of agreement between agents $a$ and $b$ on strings $\mathbf{S}$ laying on the classification boundary, $\mathbf{B} \cdot \mathbf{S}=0$. Given that $W_{a, b}=R_{a} R_{b}+Y_{a, b} \sqrt{\left(1-R_{a}^{2}\right)\left(1-R_{b}^{2}\right)}$ we only need to know $\left\{R_{a}\right\}$ and $\left\{Y_{a, b}\right\}$ to know the state of the system. 


\subsection{Update Equations}

Given a graph $\boldsymbol{G}=\left\{\{a\},\left\{\eta_{a, b}\right\}\right\}$ with vertexes $\{a\}$ and bonds $\left\{\eta_{a, b}\right\}$, the state of the system can be described by the sets of parameters $\left\{R_{a}\right\}$, defined on the vertexes and $\left\{Y_{a, b}\right\}$, defined on the bonds of $\boldsymbol{G}$. The data accessible to the agent $a$ is $\left(\sigma_{B}, \phi_{a}, \phi_{\mathbb{N}_{a}}, \mathbf{S}\right)$ where $\phi_{\mathbb{N}_{a}} \equiv\left\{\phi_{c}: c \in \mathbb{N}_{a}\right\}$. The length of such a training set is $T=\alpha_{\max } N$, which implies that $\alpha_{\max }=T / N$. For a given number $1 \leq n<T$ of examples presented to the perceptrons there is a $0<\alpha<\alpha_{\max }$ such that $n=\alpha(n) N$. Observe that, given that the minimum increment in the number of examples presented is $1, \Delta \alpha(n) \equiv \alpha(n+1)-\alpha(n)=1 / N$. By defining $\Delta t \equiv f \Delta \alpha=f / N$ and by using the update rules (2) and (6), we have that the equation for the evolution of the parameters $R_{a}$ and $Y_{a, b}$ are:

$$
\begin{aligned}
\frac{\Delta R_{a}}{\Delta t}= & \Psi_{a}\left(\beta-R_{a} \phi_{a}\right)+\lambda_{o} \sqrt{1-R_{a}^{2}} \bar{Y}_{a}+O(\Delta t) \\
\frac{\Delta Y_{a, b}}{\Delta t}= & \frac{\Psi_{a}}{\sqrt{1-R_{a}^{2}}}\left[\frac{\phi_{b}-R_{b} \beta}{\sqrt{1-R_{b}^{2}}}-Y_{a, b} \frac{\phi_{a}-R_{a} \beta}{\sqrt{1-R_{a}^{2}}}\right]-\frac{\lambda_{o} R_{a}}{\sqrt{1-R_{a}^{2}}}\left(\bar{Y}_{b}-Y_{a, b} \bar{Y}_{a}\right)+ \\
& +\mathrm{IT}_{b, a}+O(\Delta t)
\end{aligned}
$$

where $\bar{Y}_{a} \equiv M^{-1} \sum_{c} Y_{a, c}$ and $\mathrm{IT}_{b, a}$ represents a set of terms, identical to the previous ones in (13) with the indexes $a$ and $b$ interchanged.

\subsection{Perturbation}

According to Definition (9) and Eq. (12) we can expect that, if the agent $a$ learns from the rule $B$, the overlap $R_{a}=1-r_{a} / N$, where the constant $0<r_{a} \sim O(1)$. Using this hypothesis to model the action of a publicity campaign in favor of the rule $B$ and following the results reported in [13] we propose the following modification to the learning algorithm (2):

$$
\mathbf{J}_{a, n+1}=\mathbf{J}_{a, n}+\psi_{a, n} \frac{\sigma_{B, n} \mathbf{S}_{n}}{\sqrt{N}}+f_{n} \frac{\left|\mathbf{J}_{a, n}\right|}{\sqrt{N}} \sqrt{\frac{1+R_{a, n}}{N\left(1-R_{a, n}\right)}} P_{n},
$$

where the first two factors of the last term in the right-hand side of (14) are the annealing factor and the scaling factor (equivalent to (3)). The third factor is an increasing function of the overlap $R_{a}$, that produces a positive feedback in favor of the growth of $\mathbf{J}_{a}$ towards $\mathbf{B}$. Observed that this factor has an upper bound given by:

$$
\sqrt{\frac{1+R_{a}}{N\left(1-R_{a}\right)}} \leq \sqrt{\frac{2}{r_{a}}}
$$

$0 \leq P_{n}$ is a periodic function of the iteration index $n$. By considering this perturbation, Eq. (12) becomes:

$$
\frac{\Delta R_{a}}{\Delta t}=\Psi_{a}\left(\beta-R_{a} \phi_{a}\right)+\sqrt{1-R_{a}^{2}}\left[\lambda_{o} \bar{Y}_{a}+P(t)\right]+O(\Delta t)
$$

where $P(t)$ is the continuous time version of the periodic function $P_{n}$. 


\subsection{Large System Size Limit: Differential Equations}

We first consider the unperturbed set of Eqs. (12) and (13). For sufficiently large $N$ and sufficiently small $f$, the divided difference equation (12) and (13) can be transformed into differential equations. It also occurs that the components of the strings $\mathbf{S}$ are i.i.d variables with $\mathcal{P}(\mathbf{S})=\prod_{j=1}^{N}\left[\frac{1}{2} \delta_{S_{j}, 1}+\frac{1}{2} \delta_{S_{j},-1}\right]$ where $\delta_{S, X}=1$ if $S=X$ and 0 otherwise is the Kronecker delta. This stochastic character is inherited by the variables $\beta$ and $\left\{\phi_{a}\right\}$, whose joint probability, in the large $N$ limit can be estimated. In particular, for a system with only two agents we have that the joint probability is given by:

$$
\mathcal{P}\left(\beta, \phi_{a}, \phi_{b}\right)=\mathcal{N}\left(\beta \mid \Sigma_{a, b} \Lambda\left(\phi_{a}, \phi_{b}\right), \Sigma_{a, b}^{2}\right) \mathcal{N}\left(\phi_{b} \mid W_{a, b} \phi_{a}, 1-W_{a, b}^{2}\right) \mathcal{N}\left(\phi_{a} \mid 0,1\right)
$$

where $\mathcal{N}\left(x \mid \mu, \sigma^{2}\right) \equiv \exp \left(-(x-\mu)^{2} / 2 \sigma^{2}\right) / \sqrt{2 \pi \sigma^{2}}$ is a Gaussian distribution on $x$, centered at $\mu$ and with variance $\sigma^{2}$, and

$$
\begin{aligned}
\Sigma_{a, b}^{2} & \equiv \frac{\left(1-R_{a}^{2}\right)\left(1-R_{b}^{2}\right)\left(1-Y_{a, b}^{2}\right)}{1-W_{a, b}^{2}} \\
\Lambda\left(\phi_{a}, \phi_{b}\right) & \equiv \frac{\left(R_{a}-W_{a, b} \phi_{b}\right) \phi_{a}+\left(R_{b}-W_{a, b} R_{a}\right) \phi_{b}}{\sqrt{\left(1-R_{a}^{2}\right)\left(1-R_{b}^{2}\right)\left(1-Y_{a, b}^{2}\right)\left(1-W_{a, b}^{2}\right)}} .
\end{aligned}
$$

The full derivation of these expressions is presented in the appendix of [1]. For larger systems the joint probability is much harder to obtain, but it can be estimated by considering an independent bond approximation (also presented in [1]). Finally we have to consider the distribution of the parameters $\left\{R_{a}\right\}$ and $\left\{Y_{a, b}\right\}$. For most cases, the distribution of the parameters, inferred from the distribution of the strings $\mathbf{S}$, is such that the variances $\sigma_{R}^{2} \equiv\left\langle\left(R_{a}-\left\langle R_{a}\right\rangle\right)^{2}\right\rangle$ and $\sigma_{Y}^{2} \equiv\left\langle\left(Y_{a, b}-\left\langle Y_{a, b}\right\rangle\right)^{2}\right\rangle$, where $\langle x\rangle=\int \mathrm{d} x \mathcal{P}(x) x$, satisfy $\lim _{N \rightarrow \infty} \sigma_{R}^{2}=\lim _{N \rightarrow \infty} \sigma_{Y}^{2}=0$. Therefore these parameters are self-averaging [27], and in the large $N$ limit we have that $\lim _{N \rightarrow \infty}\left\langle R_{a}\right\rangle=R_{a}$ and $\lim _{N \rightarrow \infty}\left\langle Y_{a, b}\right\rangle=Y_{a, b}$.

By defining:

$$
\rho_{a, b}=\frac{1}{2}-\frac{1}{\pi} \arctan \left(\frac{R_{a}-W_{a, b} R_{b}}{\sqrt{\left(1-R_{a}^{2}\right)\left(1-R_{b}^{2}\right)\left(1-Y_{a, b}^{2}\right)}}\right)
$$

and following [1], we obtain for the vertexes and bond variables of a given graph the following set of equations:

$$
\begin{aligned}
\dot{R}_{a}= & \left(1-\sum_{c \in \mathbb{N}_{a}} \frac{\eta_{a, c}}{2}\right)\left(1-R_{a}^{2}\right)+\lambda_{o} \sqrt{1-R_{a}^{2}} \bar{Y}_{a}+ \\
& +\sum_{c \in \mathbb{N}_{a}} \frac{\eta_{a, c}}{2}\left\{\left(1-R_{a}^{2}\right) \frac{\arccos \left(Y_{a, c}\right)}{\pi}+\rho_{a, b}\left(R_{c}-W_{a, c} R_{a}\right)\right\} \\
\dot{Y}_{a, b}= & \left(1-Y_{a, b}^{2}\right) \sqrt{\frac{1-R_{b}^{2}}{1-R_{a}^{2}}} \eta_{a, b} \rho_{a, b}-\frac{\lambda_{o} R_{a}}{\sqrt{1-R_{a}^{2}}}\left(\bar{Y}_{b}-Y_{a, b} \bar{Y}_{a}\right)+\mathrm{IT}_{b, a} .
\end{aligned}
$$


Following the analysis presented in [3] we know that active bonds, i.e. those with interactions $\eta_{a, b}>0$, have parameters $Y_{a, b}$ that converge to one in a short time. By observing that:

$$
\frac{1}{2}-\lim _{Y_{a, b} \rightarrow 1} \rho_{a, b}=\Theta\left(R_{b}-R_{a}\right)
$$

and

$$
R_{c}-\lim _{Y_{a, c} \rightarrow 1} W_{a, c} R_{a}=\sin \left(\theta_{a}-\theta_{c}\right),
$$

where $\theta_{d}=\arccos \left(R_{d}\right)$, we propose the following equation for the overlaps $\left\{R_{a}\right\}$ :

$$
\begin{aligned}
\dot{R}_{a}= & \left(1-\sum_{c \in \mathbb{N}_{a}} \frac{\eta_{a, c}}{2}\right)\left(1-R_{a}^{2}\right)+ \\
& +\sqrt{1-R_{a}^{2}}\left[\sum_{c \in \mathbb{N}_{a}} \frac{\eta_{a, c}}{2} \Theta\left(R_{c}-R_{a}\right) \sin \left(\theta_{a}-\theta_{c}\right)+\lambda_{o}\right] .
\end{aligned}
$$

Observe that if all agents have, in average, the same number of connections $v \equiv$ $M^{-1} \sum_{a=1}^{M}\left|\mathbb{N}_{a}\right|$, and the interactions $\left\{\eta_{a, c}\right\}$ are drawn from a narrow distribution with mean $\eta$, the (mean field) evolution of the overlap $R_{a}$ is given by:

$$
\begin{aligned}
\dot{R}_{a}= & \frac{\eta}{2} \sqrt{1-R_{a}^{2}} \sum_{c \in \mathbb{N}_{a}} \Theta\left(R_{c}-R_{a}\right) \sin \left(\theta_{a}-\theta_{c}\right)+ \\
& +\left(1-\frac{\nu \eta}{2}\right)\left(1-R_{a}^{2}\right)+\lambda_{0} \sqrt{1-R_{a}^{2}}
\end{aligned}
$$

We observed that the right-hand-side of Eq. (24) is the combination of the inter-agent interactions in the neighborhood $\mathbb{N}_{a}$ plus an autonomous term that is minus the gradient of the potential $V\left(R_{a}\right)$ :

$$
V(R)=-\left(1-\frac{\nu \eta}{2}\right)\left(R-\frac{R^{3}}{3}\right)+\frac{\lambda_{o}}{2}\left[\arccos (R)-R \sqrt{1-R^{2}}\right]-V_{0} .
$$

It has been observed in [3] that there are four roots to the equation $\partial_{R} V(R)=0$, which are $R=-1,-R_{o}, R_{o}, 1$ where $R_{o} \equiv \sqrt{1-4(v \eta-2)^{-2} \lambda_{o}^{2}} . R=-R_{o}$ and $R=1$ are the stable points. $R=1$ is the stable point at which the system converges if the agents, at the end of the learning process classify strings in agreement with $B$, and $R=-R_{O}$ is the stable point at which the system converges if the agents classify strings mostly differently to $B$. There is a particular value of the average interaction $\eta_{o}$ such that both stable points become energetically equivalent, i.e. $V(1)=V\left(-R_{o}\right)$. By numerical calculations we found out that the bi-stability condition is satisfied when $\kappa_{o} \equiv\left(2 \lambda_{o}\right)^{-1}\left(\nu \eta_{o}-2\right)=1.12282(1)$, and thus $R_{o}=0.454754(1)$.

By preparing the system at the bi-stable regime we have that Eq. (24) become:

$$
\dot{R}_{a}=-\sin \theta_{a}\left[\lambda_{o} \kappa_{o} \sin \theta_{a}-\lambda_{o}-\frac{1+\lambda_{o} \kappa_{o}}{v} \sum_{c \in \mathbb{N}_{a}} \Theta\left(\theta_{a}-\theta_{c}\right) \sin \left(\theta_{a}-\theta_{c}\right)\right] .
$$


In a completely equivalent way we can transform the perturbed equation (16) into:

$$
\dot{R}_{a}=-\sin \theta_{a}\left[\lambda_{o} \kappa_{o} \sin \theta_{a}-\lambda_{o}-\frac{1+\lambda_{o} \kappa_{o}}{v} \sum_{c \in \mathbb{N}_{a}} \Theta\left(\theta_{a}-\theta_{c}\right) \sin \left(\theta_{a}-\theta_{c}\right)-P(t)\right]
$$

The objective of our investigation is to study the effects of an official publicity campaign (the periodic perturbation) on the opinion formation process in a community of interacting agents that initially have a liberal attitude (i.e. the agents are initially located in the basin of the stable point $-R_{o}$ ). The campaign is official because the perturbation pushes the agents' attitudes towards the official position given by $B$, on a subject where most of the agents disagree with $B$. For such a scenario we can translate the equation in overlaps (27) into an equation in phases:

$$
\dot{\theta}_{a}=\lambda_{o} \kappa_{o} \sin \theta_{a}-\lambda_{o}-\frac{1+\lambda_{o} \kappa_{o}}{v} \sum_{c \in \mathbb{N}_{a}} \Theta\left(\theta_{a}-\theta_{c}\right) \sin \left(\theta_{a}-\theta_{c}\right)-\lambda_{o} A v(\omega t)
$$

where $\theta_{a}(0) \in\left(\phi_{o}, \pi\right), \phi_{o} \equiv \arccos \left(R_{o}\right)=1.0987(1)$, and $P(t) \equiv \lambda_{o} A v(\omega t) \geq 0$ is a periodic perturbation with amplitude $\lambda_{o} A$ and frequency $\omega$. By re-scaling the time $(1+$ $\left.\lambda_{o} \kappa_{o}\right) t \rightarrow t$ and the frequency $\left(1+\lambda_{o} \kappa_{o}\right)^{-1} \omega \rightarrow \omega$ we obtain:

$$
\dot{\theta}_{a}=-\frac{1}{v} \sum_{c \in \mathbb{N}_{a}} \Theta\left(\theta_{a}-\theta_{c}\right) \sin \left(\theta_{a}-\theta_{c}\right)+\Lambda\left[\kappa_{o} \sin \theta_{a}-1-A v(\omega t)\right],
$$

where $\Lambda \equiv\left(1+\lambda_{o} \kappa_{o}\right)^{-1} \lambda_{o}$. The first term of the right-hand side of (29) is the average interaction over the neighborhood of the agent, the second term carries with the perturbation and is proportional to the rate of change of $B$.

\subsection{Solution to the Perturbed Equation (29): Schrödinger Equation for the Smallest Phase (Smallest Phase Hypothesis)}

The second term to the right-hand-side of (29) has two contributions, one autonomous and one time dependent, proportional to the constant $A$. Observe that for every neighborhood $\mathbb{N}_{a}$, there must be an agent $m$ such its phase is the smallest, i.e. $\theta_{m}^{(a)}=\min \left\{\theta_{b}: b \in \mathbb{N}_{a} \cup\{a\}\right\}$. Such an agent has a phase equation of the form:

$$
\dot{\theta}_{m}^{(a)}=\Lambda\left[\kappa_{o} \sin \theta_{m}^{(a)}-1-A v(\omega t)\right] .
$$

The associated homogeneous equation to (30) is:

$$
\dot{\varphi}=\Lambda\left[\kappa_{o} \sin \varphi-1\right],
$$

and has a solution of the form:

$$
\begin{aligned}
\tan \left(\frac{\varphi(t)}{2}\right)= & \frac{\tan \frac{\pi-\phi_{o}}{2}\left(\tan \frac{\varphi(0)}{2}-\tan \frac{\phi_{o}}{2}\right) \mathrm{e}^{\Lambda \cot \phi_{o} t}}{\left(\tan \frac{\varphi(0)}{2}-\tan \frac{\phi_{o}}{2}\right) \mathrm{e}^{\Lambda \cot \phi_{o} t}+\tan \frac{\pi-\phi_{o}}{2}-\tan \frac{\varphi(0)}{2}}+ \\
& +\frac{\tan \frac{\phi_{o}}{2}\left(\tan \frac{\pi-\phi_{o}}{2}-\tan \frac{\varphi(0)}{2}\right)}{\left(\tan \frac{\varphi(0)}{2}-\tan \frac{\phi_{o}}{2}\right) \mathrm{e}^{\Lambda \cot \phi_{o} t}+\tan \frac{\pi-\phi_{o}}{2}-\tan \frac{\varphi(0)}{2}},
\end{aligned}
$$


where $\pi-\phi_{o}$ and $\phi_{o}$ are the (stable and unstable) fixed points corresponding to $-R_{o}$ and $R_{o}$ respectively. Observe that for all initial condition $\varphi(0) \in\left(\phi_{o}, \pi\right)$ the solution to the homogeneous equation asymptotically approaches the stable point $\pi-\phi_{o}$. Observe also that the interaction term in (29) is zero only if $\theta_{a}=\theta_{m}^{(a)}$. If the interaction is not zero, and thus negative, the derivative of $\theta_{a}$ becomes negative and $\theta_{a}$ is pulled closer to the value of $\theta_{m}^{(a)}$. In consequence, if the perturbation $A v(\omega t)$ is sufficiently strong to pull the phase of agent $m$ out of the basin $\left(\phi_{o}, \pi\right)$ and into $\left(0, \phi_{o}\right)$, the other phases are attracted into the basin $\left(0, \phi_{o}\right)$ too. The hypothesis we will work with is that the agent with the smallest initial phase will keep this quality during the entire opinion formation process, i.e. $\theta_{m}^{(a)}(t) \leq \theta_{b}(t)$ for all $0 \leq t$ and $b \in \mathbb{N}_{a} \cup\{a\}$, thus, to know whether the perturbation $A v(\omega t)$ is strong enough to pull the agents into the basin of attraction of $R=1$ (the conservative basin) we only need to know if the perturbation is strong enough to pull $\theta_{m}^{(a)}$ into $\left(0, \phi_{o}\right)$.

If the $M$ agents in the population have been given initial conditions drawn randomly from a uniform distribution in $\left(\phi_{o}, \pi\right)$, it can be proven that, for sufficiently large $M$, the expected minimum phase at $t=0$ is:

$$
\begin{aligned}
\mathbb{E}\left[\theta_{m}^{(a)}(0)\right] & =\phi_{o}+\frac{1}{2 M+1} \sqrt{\frac{1+R_{o}}{2}} F_{1}\left(\frac{1}{2}, \frac{1}{2}+M, \frac{3}{2}+M ; \frac{1+R_{o}}{2}\right), \\
& =\phi_{o}+\frac{c}{M}+o\left(M^{-1}\right),
\end{aligned}
$$

where ${ }_{2} F_{1}(a, b, d ; z)$ is the Hypergeometric function [28] and

$$
c \equiv \lim _{M \rightarrow \infty} \frac{M}{2 M+1} \sqrt{\frac{1+R_{o}}{2}} F_{2}\left(\frac{1}{2}, \frac{1}{2}+M, \frac{3}{2}+M ; \frac{1+R_{o}}{2}\right) \sim O(1) .
$$

Given that the expected initial condition for the agent with the smallest phase is slightly larger than $\phi_{o}, \theta_{m}^{(a)}(0)=\phi_{o}+c M^{-1}$, and according to Eq. (32) the phase should not exceed $\pi-\phi_{o}$, we can approximate (30) by:

$$
\dot{\theta}_{m}^{(a)}=\frac{2 \Omega_{o}}{\pi-2 \phi_{o}}\left(\pi-\phi_{o}-\theta_{m}^{(a)}\right)\left(\theta_{m}^{(a)}-\phi_{o}\right)-A v(\omega t)
$$

where we have re-scaled the time and frequency such that $\Lambda t \rightarrow t$ and $\omega \Lambda^{-1} \rightarrow \omega$, and where $\Omega_{o} \equiv 2\left(\kappa_{o}-1\right)\left(\pi-2 \phi_{o}\right)^{-1}=0.260(1)$ is the characteristic frequency of the system. Since the focus of our attention will be place, from here onward, on the behavior of the minimum phase $\theta_{m}^{(a)}$, described by (34), we will drop the sub index $m$ and super-index $(a)$ from now on. By completing squares in (34) we obtain:

$$
\dot{\theta}=\frac{2 \Omega_{o}}{\pi-2 \phi_{o}}\left[\left(\frac{\pi}{2}-\phi_{o}\right)^{2}-\left(\frac{\pi}{2}-\theta\right)^{2}\right]-A v(\omega t)
$$

and by concentrating the dependency on $\theta$ on the left-hand side we have that:

$$
\frac{2 \Omega_{o} \dot{\theta}}{\pi-2 \phi_{o}}+\left(\frac{\pi \Omega_{o}}{\pi-2 \phi_{o}}-\frac{2 \Omega_{o} \theta}{\pi-2 \phi_{o}}\right)^{2}=\frac{2 \Omega_{o}\left[\kappa_{o}-1-A v(\omega t)\right]}{\pi-2 \phi_{o}} .
$$

By assuming the existence of a complex function $\psi: \mathbb{R} \rightarrow \mathbb{C}$ not zero everywhere, we can write:

$$
\left\{\frac{2 \Omega_{o} \dot{\theta}}{\pi-2 \phi_{o}}+\left(\frac{\pi \Omega_{o}}{\pi-2 \phi_{o}}-\frac{2 \Omega_{o} \theta}{\pi-2 \phi_{o}}\right)^{2}\right\} \frac{\psi(z)}{\omega^{2}}=\frac{2 \Omega_{o}\left[\kappa_{o}-1-A v(\omega t)\right]}{\pi-2 \phi_{o}} \frac{\psi(z)}{\omega^{2}},
$$


where $z \equiv \omega t$, we can identify the left-hand side of (37) with a kinetic energy term:

$$
\frac{\mathrm{d}^{2}}{\mathrm{~d} z^{2}} \psi(z)=\left\{\frac{2 \Omega_{o} \dot{\theta}}{\pi-2 \phi_{o}}+\left(\frac{2 \Omega_{o} \theta}{\pi-2 \phi_{o}}-\frac{\pi \Omega_{o}}{\pi-2 \phi_{o}}\right)^{2}\right\} \frac{\psi(z)}{\omega^{2}} .
$$

Thus, combining (37) and (38) together we obtain the Schrödinger equation of an electron in a periodic potential

$$
\mathcal{V}(z) \equiv \frac{A 2 \Omega_{o}[1-v(z)]}{\left(\pi-2 \phi_{o}\right) \omega^{2}}
$$

with total energy:

$$
\varepsilon(\omega, A) \equiv-\frac{2 \Omega_{o}\left[\kappa_{o}-1-A\right]}{\left(\pi-2 \phi_{o}\right) \omega^{2}},
$$

thus:

$$
\left[-\frac{\mathrm{d}^{2}}{\mathrm{~d} z^{2}}+\mathcal{V}(z)\right] \psi(z)=\varepsilon(\omega, A) \psi(z)
$$

Observe that Eq. (38) suggests that $\psi(z) \propto \exp \left\{\int_{t_{0}}^{t} \mathrm{~d} x[\alpha \theta(x)+\beta]\right\}$. If we take the first and second derivatives with respect to $z$ to both sides of this equation we obtain that $\psi^{\prime}(z)=$ $\omega^{-1}[\alpha \theta(t)+\beta] \psi(z)$ and $\psi^{\prime \prime}(z)=\omega^{-2}\left\{\alpha \dot{\theta}+(\alpha \theta(t)+\beta)^{2}\right\} \psi(z)$ respectively. This implies that the parameters $\alpha$ and $\beta$ must be:

$$
\alpha \equiv \frac{2 \Omega_{o}}{\pi-2 \phi_{o}}, \quad \beta \equiv-\frac{\pi \Omega_{o}}{\pi-2 \phi_{o}} .
$$

By using the expression of the first derivative of the wave function we have that the minimum phase can be expressed as:

$$
\theta(t)=\frac{\pi}{2}+\frac{\omega}{\Omega_{o}}\left(\frac{\pi}{2}-\phi_{o}\right) \frac{\mathrm{d}}{\mathrm{d} z} \ln \psi(z) .
$$

Equation (34) is a non-homogeneous Riccati equation [29], with a solution given by the expression (43), where $z=\omega t$, and $\psi(z)$ is the eigenfunction to the Schrödinger problem defined by Eq. (41) [30]. The model we proposed is such that at $t=0$ the perturbation has no impact on the opinion formation process, gradually growing afterwards, i.e. $v(0)=v^{\prime}(0)=$ 0 . By imposing boundaries $0 \leq v(t) \leq 1$ we have that $1-v(0)$ must be a maximum and thus $v_{0}^{\prime \prime} \equiv v^{\prime \prime}(0)$ must be $0<v_{0}^{\prime \prime}$. Thus, in the low frequency regime, i.e. $\omega \ll \Omega_{o}$ and for times that are below the period of $v$, i.e. $0 \lesssim t \ll \omega^{-1}$ the perturbation can be approximated by $v(\omega t)=\frac{v_{0}^{\prime \prime}}{2}(\omega t)^{2}+O\left(\omega^{3}\right)$ and Eq. (41) can be re-expressed as:

$$
\left[\frac{\mathrm{d}^{2}}{\mathrm{~d} t^{2}}+\left(\frac{\Omega_{o} A v_{0}^{\prime \prime}}{\pi-2 \phi_{o}}(\omega t)^{2}-\Omega_{o}^{2}\right)\right] \psi(t) \approx 0 .
$$

In a neighborhood of a posterior time $0<t^{\prime} \approx \omega^{-1}$ the perturbation can be expanded as $v\left(\omega\left(t^{\prime}+\tau\right)\right)=v\left(\omega t^{\prime}\right)+v^{\prime}\left(\omega t^{\prime}\right) \omega \tau+O\left(\omega^{2}\right)$. By assuming that the critical amplitude (i.e. the minimal amplitude needed to make the agents jump over the inter-basin barrier) behaves like $A_{c}(\omega)=\kappa_{o}-1+\ell\left(\pi-2 \phi_{o}\right) \Omega_{o}^{-1} \omega+O\left(\omega^{2}\right)$, the Schrödinger equation in the neighborhood of $t^{\prime}$ can be approximated by:

$$
\left[\frac{\mathrm{d}^{2}}{\mathrm{~d} \tau^{2}}-\left(1-v_{0}\right) \Omega_{o}^{2}+\left(v_{1}^{\prime} \Omega_{o}^{2} \tau-2 v_{0} \ell\right) \omega\right] \psi(\tau) \approx 0,
$$


where $v_{0} \equiv v\left(\omega t^{\prime}\right)$ and $v_{1}^{\prime} \equiv v^{\prime}\left(\omega t^{\prime}\right)$. Both Eqs. (44) and (45) can be solved by a perturbation expansion [31], proposing functions of the form $\psi=\psi_{0}+\omega^{2} \psi_{2}+O\left(\omega^{3}\right)$ for (44) and $\psi=\psi_{0}+\omega \psi_{1}+O\left(\omega^{2}\right)$ for $(45)$.

For equation (44) the perturbative solution is such that at short times the phase (43) becomes $\theta\left(t \ll \omega^{-1}\right)=\pi-\phi_{o}-L \omega^{2}$ where $0<L \sim O(1)$. This indicates that in the low frequency regime the phase becomes very close to the stable point $\pi-\phi_{o}$ (equivalent to $-R_{o}$ ) in a short time. Changes in the agents' liberal attitude are seen only at later times, when the perturbation becomes sufficiently strong. At those times we have that the equation that rules the dynamics of the system is (45), where the perturbation behaves linearly in $\omega t$. Thus by considering a perturbative expansion as a solution of (45) with the initial condition $\theta(\tau=0)=\pi-\phi_{o}$, we have that the minimum amplitude $A_{c}$ needed to take the phase $\theta$ from the stable point $\theta(\tau=0)=\pi-\phi_{o}$ to the unstable point $\theta(\tau>0)=\phi_{o}$ is:

$$
A_{c, \text { Low }}(\omega)=\left(\kappa_{o}-1\right)+\frac{v_{1}^{\prime}}{4} \frac{1}{v_{0} \sqrt{1-v_{0}}} \ln \left(\frac{1+\sqrt{1-v_{0}}}{1-\sqrt{1-v_{0}}}\right)\left(\pi-2 \phi_{o}\right) \omega+O\left(\omega^{2}\right) .
$$

At high frequencies $\Omega_{o} \ll \omega$, the number of cycles covered by the perturbation during a characteristic time of the system is $\omega \Omega_{o}^{-1} \gg 1$, thus we can substitute $v(\omega t)$ by its average over a period, i.e. $\bar{v} \equiv(2 \pi)^{-1} \int_{0}^{2 \pi} \mathrm{d} z v(z)$, in Eq. (41), thus the Schrödinger equation at high frequencies becomes:

$$
\frac{\mathrm{d}^{2}}{\mathrm{~d} z^{2}} \psi(z)=\frac{2 \Omega_{o}\left[\left(\kappa_{o}-1\right)-A \bar{v}\right]}{\omega^{2}\left(\pi-2 \phi_{o}\right)} \psi(z)
$$

with the initial condition:

$$
\phi_{o}+\frac{c}{M}=\frac{\pi}{2}+\frac{\omega\left(\pi-2 \phi_{o}\right)}{2 \Omega_{o}} \frac{\psi^{\prime}(0)}{\psi(0)} .
$$

The minimal value of the perturbation's amplitude $A_{c}$ that ensures that the phase reaches the unstable point $\phi_{o}$ at $t>0$ for high values of the frequency $\omega$ is:

$$
A_{c, \text { High }}=\frac{2 \Omega_{o}}{\bar{v}} \frac{c}{M}
$$

which depends on the value of the phase at $t=0$ but it is independent of the frequency.

Observe that the behavior of the critical amplitude at low and high frequencies, Eqs. (46) and (49) respectively, are such that no interpolation to intermediate values of the frequency are meaningful.

\subsection{Particular Case $v(\omega t)=\sin ^{2}(\omega t / 2)$}

As an illustrative example we study in this section the perturbation $v(\omega t)=\sin ^{2}\left(\frac{\omega t}{2}\right)$ that produces the Schrödinger equation (41) that can be transformed into Mathieu's Equation $\psi^{\prime \prime}(x)+[a-2 q \cos (2 x)] \psi(x)=0[28,32]$, with variable $x=\omega t / 2$ and parameters $a \equiv$ $4 \Omega_{o}\left[A-2\left(\kappa_{o}-1\right)\right]\left[\left(\pi-2 \phi_{o}\right) \omega^{2}\right]^{-1}$ and $q \equiv 2 \Omega_{o} A\left[\left(\pi-2 \phi_{o}\right) \omega^{2}\right]^{-1}$. The general solution to the Mathieu's Equation can be expressed as a linear combination of even $\mathscr{M}_{c}(a, q ; x)$ and odd $\mathscr{M}_{s}(a, q ; x)$ Mathieu's functions [28], such that $\psi(x)=C_{c} \mathscr{M}_{c}(a, q ; x)+C_{s} \mathscr{M}_{s}(a, q ; x)$. Given that the equation of the phase (34) is of the first order, we expect the solution to present only one free constant (that can be adjusted through the particular initial conditions). Thus, from Eq. (48) we have that:

$$
\phi_{o}+\frac{c}{M}=\frac{\pi}{2}+\frac{\omega\left(\pi-2 \phi_{o}\right)}{2 \Omega_{o}} \frac{C_{s} \mathscr{M}_{s}^{\prime}(a, q ; 0)}{C_{c} \mathscr{M}_{c}(a, q ; 0)}
$$


$A<A C$

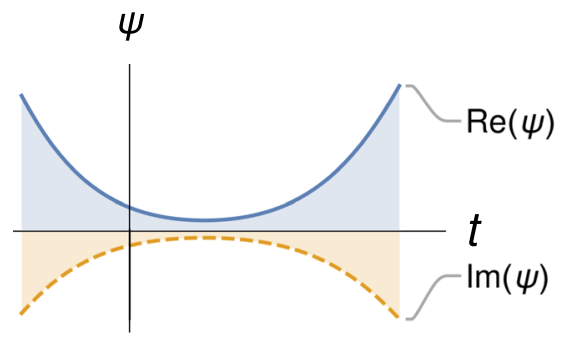

$A \geq A C$

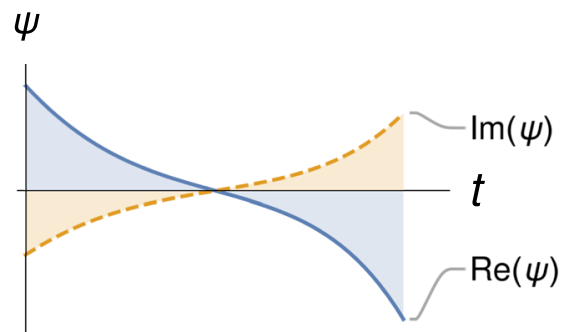

Fig. 1 Real and Imaginary parts of the system's wave function $\psi(z)$ for values of the amplitude $A$ bellow (left panel) and above (right panel) $A_{C}$

where we have used that $\mathscr{M}_{s}(a, q ; 0)=\mathscr{M}_{c}^{\prime}(a, q ; 0)=0$ (both are odd functions). Thus

$$
\frac{C_{s}}{C_{c}}=-\frac{\Omega_{o}}{\omega} \frac{\mathscr{M}_{c}(a, q ; 0)}{\mathscr{M}_{s}^{\prime}(a, q ; 0)}\left(1-\frac{2}{\pi-2 \phi_{o}} \frac{c}{M}\right),
$$

and thus the wave function of the problem becomes:

$$
\begin{aligned}
\psi(x)= & \frac{C_{c}}{\mathscr{M}_{s}^{\prime}(a, q ; 0)}\left[\mathscr{M}_{s}^{\prime}(a, q ; 0) \mathscr{M}_{c}(a, q ; x)\right. \\
& \left.-\frac{\Omega_{o}}{\omega}\left(1-\frac{2}{\pi-2 \phi_{o}} \frac{c}{M}\right) \mathscr{M}_{c}(a, q ; 0) \mathscr{M}_{s}(a, q ; x)\right],
\end{aligned}
$$

where the primes indicate derivation with respect to $x$. Following Eq. (43) we have that

$$
\begin{aligned}
& \theta(t ; \omega, A)=\frac{\pi}{2}+\frac{\omega}{\Omega_{o}} \frac{\pi-2 \phi_{o}}{2} \\
& \times \frac{\mathscr{M}_{s}^{\prime}(a, q ; 0) \mathscr{M}_{c}^{\prime}(a, q ; x)-\frac{\Omega_{o}}{\omega}\left(1-\frac{2}{\pi-2 \phi_{o}} \frac{c}{M}\right) \mathscr{M}_{c}(a, q ; 0) \mathscr{M}_{s}^{\prime}(a, q ; x)}{\mathscr{M}_{s}^{\prime}(a, q ; 0) \mathscr{M}_{c}(a, q ; x)-\frac{\Omega_{o}}{\omega}\left(1-\frac{2}{\pi-2 \phi_{o}} \frac{c}{M}\right) \mathscr{M}_{c}(a, q ; 0) \mathscr{M}_{s}(a, q ; x)}
\end{aligned}
$$

where $a$ and $q$ are functions of the parameters of the system. There exists a set $\mathbb{A}_{\omega}$ of amplitudes that make the perturbation sufficiently strong to make the phase reach the unstable point $\phi_{o}$ at a posterior time $t_{o}$, i.e. $\mathbb{A}_{\omega}=\left\{A \in \mathbb{R}: \exists t_{o}\right.$ such that $\left.\theta\left(t_{o} ; \omega, A\right)=\phi_{o}\right\}$. The critical amplitude as a function of the frequency $\omega$ is $A_{c}(\omega)=\min \mathbb{A}_{\omega}$. We observed that for values of $A<A_{c}$ the Schrödinger's wave function is different from zero for all $0<t$, whereas for $A \geq A_{c}$, there exists $0<t_{o}^{\prime}$ such that $\psi\left(\omega t_{o}^{\prime}\right)=0$ and $\psi^{\prime}\left(\omega t_{o}^{\prime}\right)<0$ (Fig. 1), which implies that the ratio $\psi^{\prime}(t) / \psi(t)$ becomes large and negative for $t \rightarrow t_{o}^{\prime}$, and from Eq. (53) we have that $\theta \rightarrow \phi_{0}$. We also observe that the solution of the Mathieu's equation $\psi(z) \in \mathbb{C}$, is such that $\Im(\psi(z)) / \Re(\psi(z))=C$ independent of $z$, thus $\psi^{\prime}(z) / \psi(z) \in \mathbb{R}$, thus $\theta(t ; \omega, A)$, as expressed in (53), is real for all $t$.

By analyzing the eigenvalue of the Schrödinger's equation (41) at the critical amplitude $\varepsilon_{c}(\omega) \equiv 2 \Omega_{o}\left[A_{c}(\omega)-\left(\kappa_{o}-1\right)\right]\left[\left(\pi-2 \phi_{o}\right) \omega^{2}\right]^{-1}$, we observe that for sufficiently low frequencies the critical amplitude (46) is such that $\varepsilon_{c}\left(\omega \ll \Omega_{o}\right)>0$ and for sufficiently high frequencies and sufficiently large systems, i.e. $O(1) \sim 4 c\left[\left(\pi-2 \phi_{o}\right) \bar{v}\right]^{-1}<M$ which is a very mild assumption, the critical amplitude (49) is such that $\varepsilon_{c}\left(\omega \gg \Omega_{o}\right)<0$. Therefore 
we define the critical frequency of the system $\omega_{c}$ the frequency at which the eigenvalue of the Schrödinger equation becomes zero, i.e. $A_{c}\left(\omega_{c}\right)=\kappa_{o}-1$.

\section{Numerical Results}

To obtain the perturbation's critical amplitude we performed a numerical integration of systems of differential equations (30), with sizes $M=5,10,15,20,25,30,35,40$, and a sinusoidal perturbation $\sin ^{2}(\omega t / 2)$. To validate the smallest phase hypothesis the system is constructed in a way that the $M$ agents form a unique cluster and no agent is disconnected. By applying a second order Runge-Kutta method we integrated the trajectories in the intervals $t \in(0,10 \pi / \omega)$, where $\omega$ is the frequency of the perturbation. The agents were assigned initial phases $\theta_{a}(0)$ drawn from a flat distribution $\theta_{a}(0) \in\left(\phi_{o}, \pi\right)$, and the critical amplitude was found by applying a bisection method. The results are presented in Fig. 2. The first feature we observe from these curves is that all collide to the same curve for small values of the frequency $\omega \ll \Omega_{o}$. The linear, least-square fit of the data $A_{c \text {.Low }}(\omega)=A_{0}+A_{1} \omega$ produces an intersect $A_{0}=0.123(1)$ indistinguishable from $\left(\kappa_{o}-1\right)$ and a slope $A_{1}=2.379(1)$ that, by applying Eq. (46) corresponds to a time $t^{\prime}=1.074(1) \omega^{-1}$. Both results are consistent with Eq. (46) and with assumption $t^{\prime} \approx \omega^{-1}$ leading to Eq. (45). Observe that the range of frequencies covered in Fig. 2 is bellow $\Omega_{o}$. We did not managed to obtain meaningful results for the high frequency regime, due to the technical difficulty associated to finding zeros of highly oscillating functions. Even so, the numerical analysis of the results presented a tendency $A_{c, \text { High }} \sim O\left(M^{-0.7(3)}\right)$ which is consistent with Eq. (49).

Observe that the error bars for the measurement of the critical amplitude at low frequencies are negligible compared to the error bars associated to equivalent measurements at high frequencies. Error bars were computed by integrating 100 realizations of each system of differential equations (29). For high-frequencies the estimated error associated to each data point becomes one order of magnitude less than the amplitude $A_{c}$ itself $\left[O\left(10^{-1} A_{c}\right)\right]$, whereas for the low-frequency regime, the error associated to each data point is negligible. The difference in behavior is due to the fact that for higher frequencies the perturbation effectively acts at very short times, $t \ll \Omega_{o}^{-1}$, thus the noise introduce through the initial conditions has an impact in the results. At low values of the perturbation frequency all phases have time to relax towards the stable point $\pi-\phi_{o}$, thus for the time when the perturbation is strong enough to produce changes in the system $\left(\Omega_{o}^{-1} \ll t^{\prime}\right)$, all phases are almost identical $\theta_{a}\left(t^{\prime}\right)=\pi-\phi_{o}-\varepsilon_{a}$, with $0<\varepsilon_{a} \sim O\left(10^{-6}\right)$. Thus the estimate of the variance computed from different realizations of the system is almost negligible.

To validate the hypothesis that the agent with the minimum phase remains unchanged over the entire process, we compare the numerical calculations with $M=20,30$, and 40 presented in Fig. 2 with the critical amplitude obtained by numerically solving the equation $\theta\left(\tilde{t} ; \omega, A_{c}\right)=\phi_{o}$, where $\theta\left(\tilde{t} ; \omega, A_{c}\right)$ is given by Eq. (53), and $A_{c}$ is the minimal amplitude for which a $\tilde{t}<\infty$ exists. The results are presented in Fig. 3 . The match between the results obtained by the numerical integration of the system (29) and the application of the analytical solution (53) are indistinguishable for sufficiently small values of the frequency $\omega \ll \Omega_{o}$. For the intermediate $\left(\omega \sim \Omega_{o}\right)$ and high frequency $\left(\omega \gg \Omega_{o}\right)$ regimes the match is not as good, although the qualitative behavior of the curves is alike. We believe that the source of the discrepancy is the above mentioned technical difficulty of finding the zeros of highly oscillating functions. These results give support to the hypothesis and approximations used to derive expression (53). 


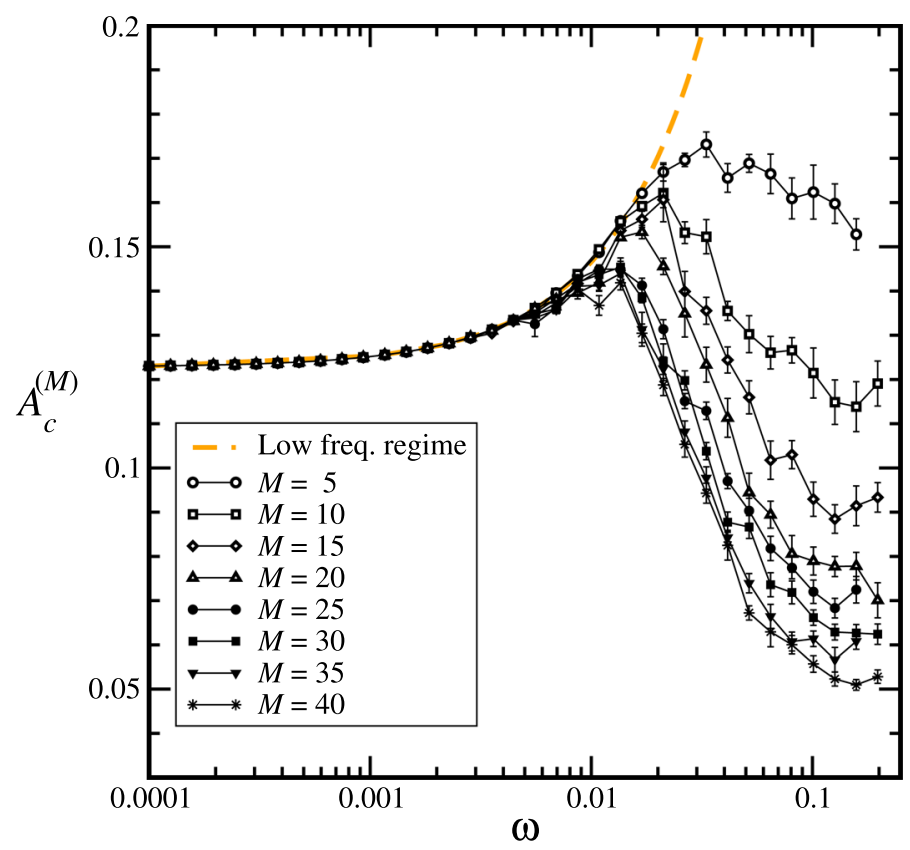

Fig. 2 Critical amplitude of the perturbation as a function of the frequency, for systems with sizes $M=$ $5,10,15,20,25,30,35,40$. The curves were obtained by integrating systems of differential equations, with perturbations of the form $A \sin ^{2}(\omega t / 2)$. The dashed line represents the best fit for the low-frequency regime $A_{c}(\omega)=0.123(1)+2.379(1) \omega$

We have observed that in the high frequencies $\left(\omega \gg \Omega_{c}\right)$ regime the critical amplitude depends on the size of the system through the initial conditions. Thus, we computed the curve $\varepsilon_{c}(\omega)$ [Eq. (40)] for systems sizes $M=10,50,100,150,300,1000$. The result of this computation is presented in Fig. 4. In the inset of Fig. 4 we present the solution of the equation $\varepsilon_{c}\left(\omega_{c}\right)=0$ as a function of $M$. We observe that the critical frequency depends on the size of the system as $\omega_{c}(M)=1.75(1)\left[1+1.13(1) M^{\frac{1}{2}}\right]^{-1}$. This result indicates that the low frequency region becomes negligibly small for large values of $M$.

\section{Discussion}

We proposed a model of opinion formation in societies of adaptive agents where there is a set of rules $B$ that determined what is socially acceptable. In the present work we allow $B$ to adjust according to the average position of the population with a constant of proportionality $\lambda_{o}$, and we have also introduced a periodic perturbation that mimics the action of a publicity campaign in favor of $B$. The effectiveness of periodic publicity campaigns has been analyzed at length in [13], where a multi-channel approach is studied. Although we limit our approach to the single channel case, the use of a periodic function to model the publicity campaign is well based. By the application of statistical mechanics techniques we constructed a description of the system based on a set of differential equations ruling the evolution of the parameters $\left\{R_{a}\right\}$, that represent the agreement of the agents $\{a\}$ with $B$. For this system there are only two stable fixed points, dubbed the conservative point $R=1$, and the liberal point $R=$ 


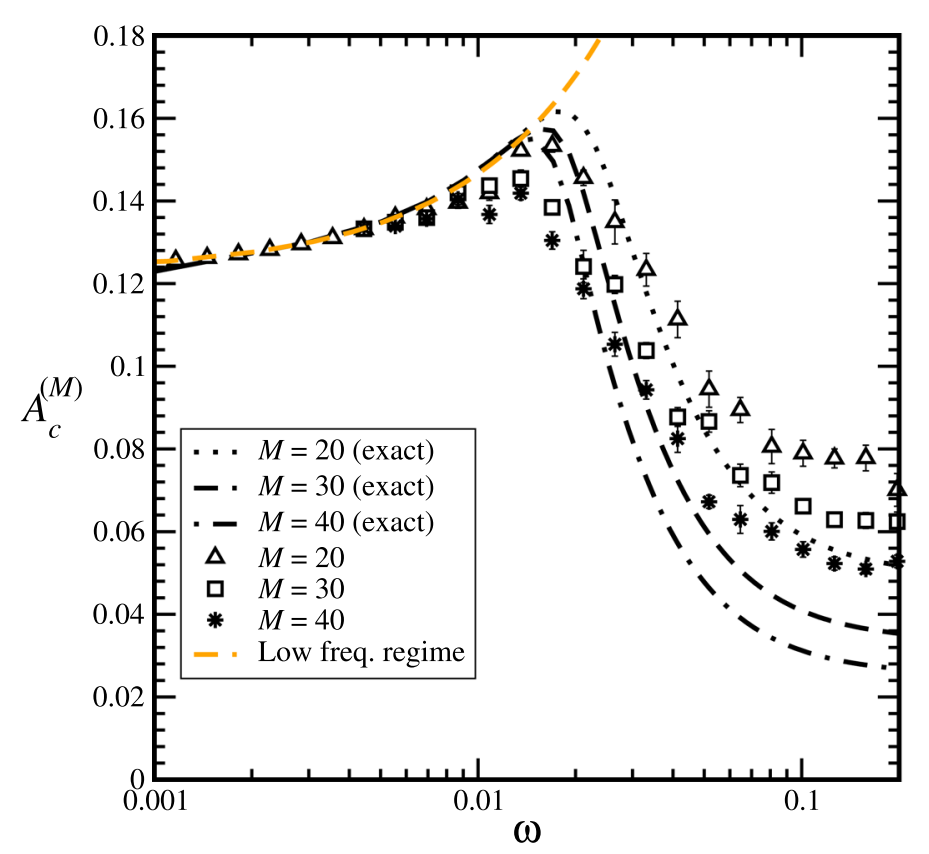

Fig. 3 Critical amplitude $A_{C}$ as a function of the frequency $\omega$ for systems with $M=20,30$, and 40 agents. The symbols corresponds to numerical integration of the system of Eq. (24), whereas the continuous curves correspond to the numerical calculation of $A_{c}$ using Eq. (53). It can be observed that for sufficiently small values of the frequency curves and symbols overlap, whereas the quantitative discrepancies observed for larger values of the frequency can be associated with the technical difficulties of finding zeros of highly oscillating functions

$-\sqrt{1-4(v \eta-2)^{-2} \lambda_{o}^{2}}$ where $v$ is the average number of neighbors and $\eta$ is the average social strength.

By imposing mild conditions on the perturbation $v(z)$, i.e. $v$ is twice differentiable and bounded, we managed to reduce the the analysis of the system of differential equations (29) to the analysis of the equation (30) that rules the evolution of the smallest phase $\theta_{m}^{(a)}=\min \left\{\theta_{a} \equiv\right.$ $\arccos \left(R_{a}\right)$ \}. By applying a quadratic approximation to (30) we obtained the Riccati equation (34), which admits an exact solution (43). Such a solution is linked to the solution of the Schrödinger equation (41) that describes the behavior of an electron in a periodic potential. By exploring the behavior of the solution of the Schrödinger equation (41) for values of the perturbation's frequency $\omega$ much larger (smaller) than the characteristic frequency of the system $\Omega_{o}=0.260(1)$, we estimated the value of the minimum perturbation's amplitude $A_{c}$ needed to move agents with liberal attitude [i.e. with phases $\theta$ in the basin $\left(\phi_{o}, \pi\right)$ ] into the conservative basin $\left(0, \phi_{o}\right)$, as a function of $\omega$. We observed that for very low frequencies, the critical amplitude $A_{c \text {,Low }}(\omega)$ is a linear function of $\omega$, Eq. (46), whereas for high values of $\omega$ the critical amplitude strongly depends on the initial conditions $\theta_{m}^{(a)}(0)$. Given that the initial conditions of the system with $M$ agents are drawn from a uniform distribution in the interval $\left(\phi_{o}, \pi\right)$, the expected value of the minimum phase is $\phi_{o}+c M^{-1}$ with $c \sim O(1)$. Thus, we have obtained that $A_{c, \mathrm{High}} \propto M^{-1}$.

To validate our results we performed a number of numerical integration of the set of Eq. (29), for system sizes $M=5,10,15,20,25,30,35$, 40, and for a periodic perturbation of the form $v(z)=\sin ^{2}(z)$. For this particular case, the Schrödinger equation (41) is linked 


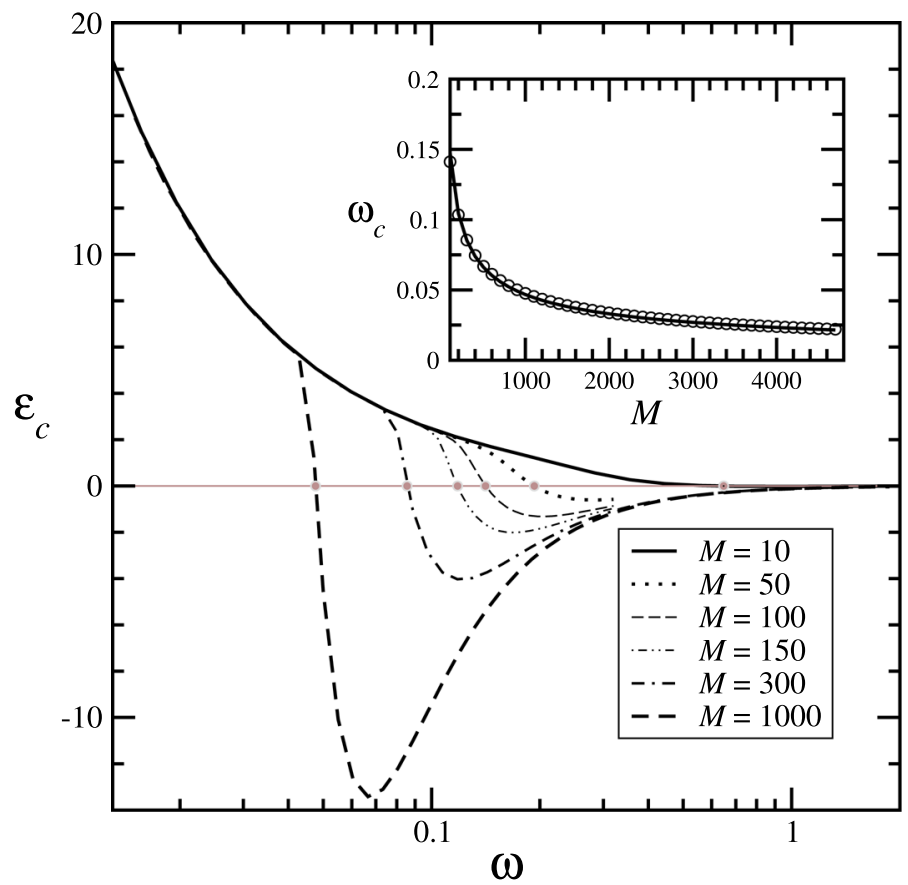

Fig. 4 Schrödinger eigenvalue $\varepsilon_{c}(\omega)$ as a function of the frequency for system sizes $M=$ $10,50,100,150,300,1000$. In the inset we present the value of the critical frequency $\omega_{c}$ as a function of the system size. The full line represents the best fit $\omega_{c}(M)=1.75(1)\left[1+1.13(1) M^{\frac{1}{2}}\right]^{-1}$

to Mathieu's equation $\psi^{\prime \prime}(x)+[a-2 q \cos (2 x)] \psi(x)=0$, with variable $x=\omega t / 2$ and parameters $a \equiv 4 \Omega_{o}\left[A-2\left(\kappa_{o}-1\right)\right]\left[\left(\pi-2 \phi_{o}\right) \omega^{2}\right]^{-1}$ and $q=2 \Omega_{o} A\left[\left(\pi-2 \phi_{o}\right) \omega^{2}\right]^{-1}$. The numerical results obtained are presented in Figs. 2 and 3, which are consistent with the expressions obtained from the analysis of the equation of the smallest phase (30).

Our model is closed related to the scenario presented in [33], where the influence of a mass communication anti-marijuana campaign in adolescents is analyzed. In our model, $B$ represents the position of the government, the interacting agents are school attending teenagers, and the social issue opinions are formed over is the use of marijuana. It was found that exposure to social interaction about campaign messages can affect behavior, and such interactions are favored if the tone of the campaign is not perceived as oppressive (low $A$ in our model) and the ads are not 'infrequent' (above the characteristic frequency $\Omega_{o}$ in our model). These results are consistent with the smallest phase hypothesis and the highfrequency low-amplitude strategy of our findings.

In summary, our model indicates that if the government desires to regularly perturb the population of voters with publicity campaigns, it is more profitable (for the government) to do so with a frequency higher than the characteristic frequency of the system $\Omega_{o}$. In doing so, the amplitude of the oscillation decays with the size of the population $A_{c \text {, High }} \propto M^{-1}$, whereas for low frequencies $\omega \ll \Omega_{o}$ the amplitude is always larger than a minimum value $A_{c \text {, Low }}>$ $\kappa_{o}-1$. 
Acknowledgements The author would like to acknowledge the constructive discussions with Dr. R. C Alamino and Dr I. Yurkevich. The advise of Dr. C. M Juarez is kindly appreciated.

Open Access This article is licensed under a Creative Commons Attribution 4.0 International License, which permits use, sharing, adaptation, distribution and reproduction in any medium or format, as long as you give appropriate credit to the original author(s) and the source, provide a link to the Creative Commons licence, and indicate if changes were made. The images or other third party material in this article are included in the article's Creative Commons licence, unless indicated otherwise in a credit line to the material. If material is not included in the article's Creative Commons licence and your intended use is not permitted by statutory regulation or exceeds the permitted use, you will need to obtain permission directly from the copyright holder. To view a copy of this licence, visit http://creativecommons.org/licenses/by/4.0/.

\section{References}

1. Neirotti, J.: Anisotropic opinion dynamics. Phys. Rev. E 94, 012309 (2016)

2. Neirotti, J.: Consensus formation times in anisotropic societies. Phys. Rev. E 95, 062305 (2017)

3. Neirotti, J.: Anisotropic opinion dynamics with an adaptive social rule. Phys. Rev. E 98, 052306 (2018)

4. Nardini, C., Kozma, B., Barrat, A.: Who's talking first? Consensus or lack thereof in coevolving opinion formation models. Phys. Rev. Lett. 100, 158701 (2008)

5. Shao, J., Havlin, S., Stanley, H.E.: Dynamic opinion model and invasion percolation. Phys. Rev. Lett. 103, 018701 (2009)

6. Török, J., Iñíguez, G., Yasseri, T., San Miguel, M., Kaski, K., Kertész, J.: Opinions, conflicts, and consensus: modeling social dynamics in a collaborative environment. Phys. Rev. Lett. 110, 088701 (2013)

7. Pinheiro, F., Santos, M.D., Santos, F.C., Pacheco, J.: Origin of peer influence in social networks. Phys. Rev. Lett. 112, 098702 (2014)

8. Nicosia, V., Skardal, P.S., Arenas, A., Latora, V.: Collective phenomena emerging from the interactions between dynamical processes in multiplex networks. Phys. Rev. Lett. 118, 138302 (2017)

9. Baumann, F., Lorenzo-Spreen, P., Sokolov, I.M., Starnini, M.: Modeling echo chambers and polarization dynamics in social networks. Phys. Rev. Lett. 124, 048301 (2020)

10. Moore, T., Finley, P., Brodsky, N., Brown, T., Apelberg, B., Ambrose, B., Glass, R.: Modeling education and advertising with opinion dynamics. J. Artif. Soc. Social Simul. 18, 7 (2015)

11. Gupta, A., Moharir, S., Sahasrabudhe, N.:influencing opinion dynamics in networks with limited interaction. arXiv:2002.00664v [cs.SI] (2020)

12. Carletti, T., Fanelli, D., Grolli, S., Guarino, A.: How to make an efficient propaganda. Europhys. Lett. 74, 222 (2006)

13. Eshghi, S., Preciado, V., Sarkar, S., Venkatesh, S.S., Zhao, Q., D’Souza, R., Swami, A.: Spread, then target, and advertise in waves: optimal budget allocation across advertising channels. IEEE Trans. Net. Sci. Eng. 1, 99 (2018)

14. Stewart, H., Mason, R.: EU referendum: $£ 9$ m taxpayer-funded publicity blitz pushes case to remain. The Guardian, 7th April (2016)

15. Deffuant, G., Neau, D., Amblard, F., Weisbuch, G.: Mixing beliefs among interacting agents. Adv. Complex Syst. 3, 87 (2000)

16. Engel, A., Van den Broeck, C.: Stat. Mech. Learn. CUP, Cambridge (2001)

17. Kacperski, K., Holyst, J.A.: Phase transitions and hysteresis in a cellular automata-based model of opinion formation. J. Stat. Phys. 84, 169 (1996)

18. Hebb, D.O.: The Organization of Behavior. Wiley, New York (1949)

19. Bishop, C.M.: Neural Networks for Pattern Recognition. Oxford University Press, Oxford (1995)

20. Caticha, N., Kinouchi, O.: Time ordering in the evolution of information processing and modulation systems. Philos. Mag. B 77, 1565 (1998)

21. Baron, R.S., Hoppe, S.I., Kao, C.F., Brunsman, B., Linneweh, B., Rogers, B.: Social corroboration and opinion extremity. J. Exp. Social Psychol. 32, 537 (1996)

22. Lord, C.G., Ross, L., Lepper, M.R.: J. Personal. Soc. Psychol. 37, 2098 (1979)

23. Gilbert, E., Bergstrom, T., Karahalios, K.: Proceedings of the Hawaii International Conference on System Sciences. In: Sprague, R.J. (ed.) IEEE Computer Society, Washington DC, 1 (2009)

24. Caticha, N., Vicente, R.: Agent-based social psychology: from neurocognitive processes to social data. Adv. Complex Syst. 14, 711 (2011)

25. Latané, B.: The psychology of social impact. Am. Psychol. 36, 343 (1981) 
26. Lewenstein, M., Nowak, A., Latané, B.: Statistical mechanics of social impact. Phys. Rev. A 45, 763 (1992)

27. Reents, G., Urbanczik, R.: Self-averaging and on-line learning. Phys. Rev. Lett. 80, 5445 (1998)

28. Abramowitz, M., Stegun, I.: Handbook of Mathematical Functions with Formulas, Graphs, and Mathematical Tables. Dover Publications, New York (1984)

29. Ince, E.L.: Ordinary Differential Equations, pp. 23-25. Dover Publications, New York (1956)

30. Mermin, N.D., Ashcroft, N.: Solid State Physics. Academic Press, Cambridge (1976)

31. Merzbacher, E.: Quantum Mechanics. Wiley, New York (1998)

32. Morse, P.M., Feshbach, H.: Methods of Theoretical Physics. McGraw-Hill, New York (1953)

33. David, C., Capella, J.N., Fishbain, M.: The social diffusion of influence among adolescents: group interaction in a chat room environment about antidrug advertisements. Commun. Theory 16, 118 (2006)

Publisher's Note Springer Nature remains neutral with regard to jurisdictional claims in published maps and institutional affiliations. 